\title{
Cellular uptake of nanoparticles: Involvement of caveolae?
}

\author{
Tore Skotland ${ }^{\mathrm{a} *}$, Tore Geir Iversen, ${ }^{\mathrm{a}}$ and Kirsten Sandviga, \\ ${ }^{a}$ Department of Molecular Cell Biology, Institute for Cancer Research, Oslo University Hospital, \\ The Norwegian Radium Hospital, Oslo, Norway \\ ${ }^{b}$ Department of Biosciences, University of Oslo, Oslo, Norway
}

Submitted: March 31, 2021

Accepted: April 14, 2021

Published: April 15, 2021

\section{ABSTRACT}

Here we discuss some pitfalls and challenges briefly when investigating which endocytic mechanisms are involved in the cellular uptake of nanoparticles. We specifically discuss some common misunderstandings regarding studies claimed to demonstrate uptake via caveolae. Scientists in the nanomedicine field should be aware that reducing the membrane content of cholesterol by adding methyl- $\beta$-cyclodextrin removes caveolae and inhibits other uptake mechanisms, such as macropinocytosis as well. Furthermore, the general tyrosine kinase inhibitor genistein is not a specific inhibitor of uptake from caveolae. Moreover, one can still see that scientists in the field write that they want to direct transport of their particles to caveolae and caveosomes to avoid lysosomal degradation. However, caveosomes are artifacts caused by overexpression of caveolin-1 constructs, and ligands or particles taken up by caveolae are transported to endosomes and lysosomes as reported for other types of endocytosis.

\section{KEYWORDS:}

Nanoparticles, Cellular Uptake, Endocytosis, Caveolae, Caveosomes, Inhibitors, Methyl- $\beta$ cyclodextrin, Genistein

\section{RATIONALE AND PURPOSE}

We here focus on some common misunderstandings and pitfalls concerning the uptake of nanoparticles via caveolae. Specifically, we warn against drawing conclusions based on studies using unspecific pharmacological inhibitors, and we remind scientists that "caveosomes" turned out to be artifacts. We hope this article will improve the quality of reports in the nanoparticle field.

\section{INTRODUCTION}

Nanoparticles (NPs) are taken up into mammalian cells by different endocytic mechanisms. Such mechanisms are cell typedependent and also regulated by other growth conditions such as cell density, polarity, and presence of growth factors. The complexity of mechanisms has been increasing as more molecular details are revealed, and it is not straightforward to study which uptake mechanisms are involved [1-3]. However, there are some general misunderstandings that need more attention. Therefore, ten years ago, we published an article aiming to help NP researchers with these challenges, including publishing a toolbox of pharmacological inhibitors that are used in such studies [1]. Although that review entitled "Endocytosis and intracellular transport of nanoparticles: Present knowledge and need for future studies" is now cited almost 800 times, the need to improve the quality of studies of endocytosis of NPs is still nearly as described ten years ago. We already in that review discussed the challenges when concluding if the cellular uptake of NPs was via caveolae and that caveosomes were artifacts due to overexpression of caveolin-1 constructs. Even though this message has also been given in several articles aiming at the endocytic community (e.g., $[2,4])$ and has been presented at every CLINAM meeting (and other meetings) during the last ten years, one can still often see discussions involving caveosomes. We hope that a short commentary aimed at

* Corresponding author: toresko@uio.no 
reaching the NP community in an open-access journal and focusing on this single subject should help authors and reviewers improve the quality of studies describing the endocytosis of nanoparticles and stop using the misconception of caveosomes. This story about caveosomes in NP research shows similarities with two recent articles about misconceptions that may persist in literature for a long time and referred to as "unicorns," i.e., mythical creatures with no basis in reality $[5,6]$.

\section{DISCUSSION}

\section{Caveolae and caveosomes}

Caveolae (meaning "little caves") are flaskformed structures of $50-80 \mathrm{~nm}$ on the cell surface; in some cells (e.g., adipocytes, endothelial cells, and muscle cells), they can be present at high density and cover up to $50 \%$ of the plasma membrane area, whereas other cells contain only a few or no caveolae $[7,8]$. The function of these structures has been discussed for many years. Are they active in endocytosis or mostly stable structures contributing little to endocytic uptake [9]? Caveolae seem to play an important role in uptake and transcytosis of albumin in endothelial cells, where albumin binds to the receptor gp60 (also called albondin) [10]. This receptor has also been described to be involved in the transport of the active drug (paclitaxel bound to albumin) in Abraxane $^{\mathbb{B}}$ [11]. However, there is still not much evidence that caveolae are involved in a similar mechanism for uptake and intracellular vesicle formation in other cell types. In fact, caveolae seem to be rather stable structures not giving major contributions to the cellular endocytic uptake [7, 12]. Approximately 20 years ago, caveolae were reported to be involved in cellular uptake of the SV40 virus, and it was published that the virus was found in intracellular vesicles called caveosomes; the content of these vesicles was stated to be transported to the Golgi apparatus and the endoplasmic reticulum, and thereby escape lysosomal degradation [13]. Some years later, the same authors reported that SV40 was taken up even more efficiently by clathrin- and caveolin-independent endocytosis [14]. In 2010 they reported that caveosomes were artifacts obtained by overexpression of constructs of caveolin-1 and that the term caveosomes no longer should be used [15]. If NPs are taken up via caveolae, they are first transported to early endosomes, similarly as described for other endocytic mechanisms (Figure 1).

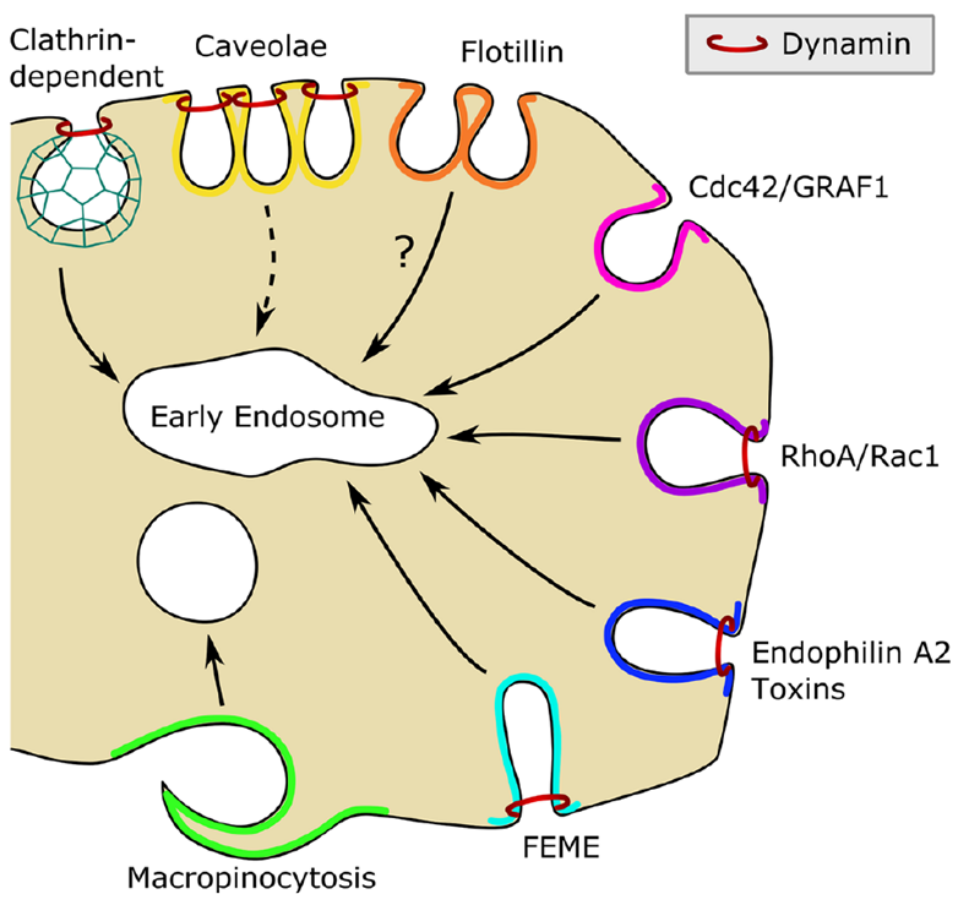

Figure 1. An overview of endocytic mechanisms in a non-polarized cell showing that all internalized vesicles, with the possible exception of the vesicles formed by micropinocytosis, go to early endosomes. For a discussion of these uptake mechanisms, see [2]. 
It should be noted that caveolae during recent years have been shown to have several cellular functions. A recently described function for caveolae is their ability to provide additional membrane upon mechanical stress and reform in an ATP-dependent manner [8], which also may explain the high density of caveolae in some cell types $[7,16,17]$.

\section{Studies concluding that NPs are taken up} via caveolae

As discussed earlier [1], it is not straightforward to study which endocytic uptake mechanisms are involved in cellular uptake. Most pharmacological inhibitors are not specific, and inhibition of one pathway may lead to compensation with higher endocytic uptake via other pathways [1]. One common mistake is that reduced endocytosis following the treatment of cells with methyl- $\beta$ cyclodextrin to extract cholesterol demonstrates that the endocytic uptake is via caveolae. Although it is correct that such treatment can extract cholesterol from the plasma membrane, including caveolae, extraction of cholesterol may also affect other endocytic processes such as clathrin-dependent uptake [18] and macropinocytosis [19]. In addition to the use of methyl- $\beta$-cyclodextrin, also other agents, like filipin (interacting with cholesterol) or statins (lowering cholesterol synthesis), will affect various endocytic processes, discussed in [1, 20, 21]. In fact, we are not aware of any endocytic process that has been demonstrated to be unaffected following reduction or removal of cholesterol.

Also, reduced cellular uptake of NPs following treatment of cells with genistein is often reported to demonstrate uptake via caveolae. It should, however, be noted that although genistein may inhibit uptake from caveolae, e.g., inhibit SV-40 induced vesicle formation from caveolae [22], genistein is a general inhibitor of tyrosine kinases and will therefore affect other uptake processes activated by tyrosine kinases. This includes uptake of receptors that need tyrosine phosphorylation for accumulation in clathrincoated pits, such as the EGF receptor, and macropinocytosis activated by the EGF receptor (see [1] for a more extensive discussion).

Colocalization of NPs with cholera toxin is often taken as evidence for uptake via caveolae. However, cholera toxin is taken up via several endocytic mechanisms [23-25], and colocalization between NPs and cholera toxin can therefore not be taken as evidence for uptake of NPs via caveolae. One should also remember that observation of NPs within a given structure such as caveolae does not mean that the NPs are taken up that way; the NPs may simply be stuck in that structure. It may also be a challenge to decide whether a structure looking like an internalized vesicle with the size of caveolae in an EM image, in reality, is internalized or connected to the surface (Figure 2).

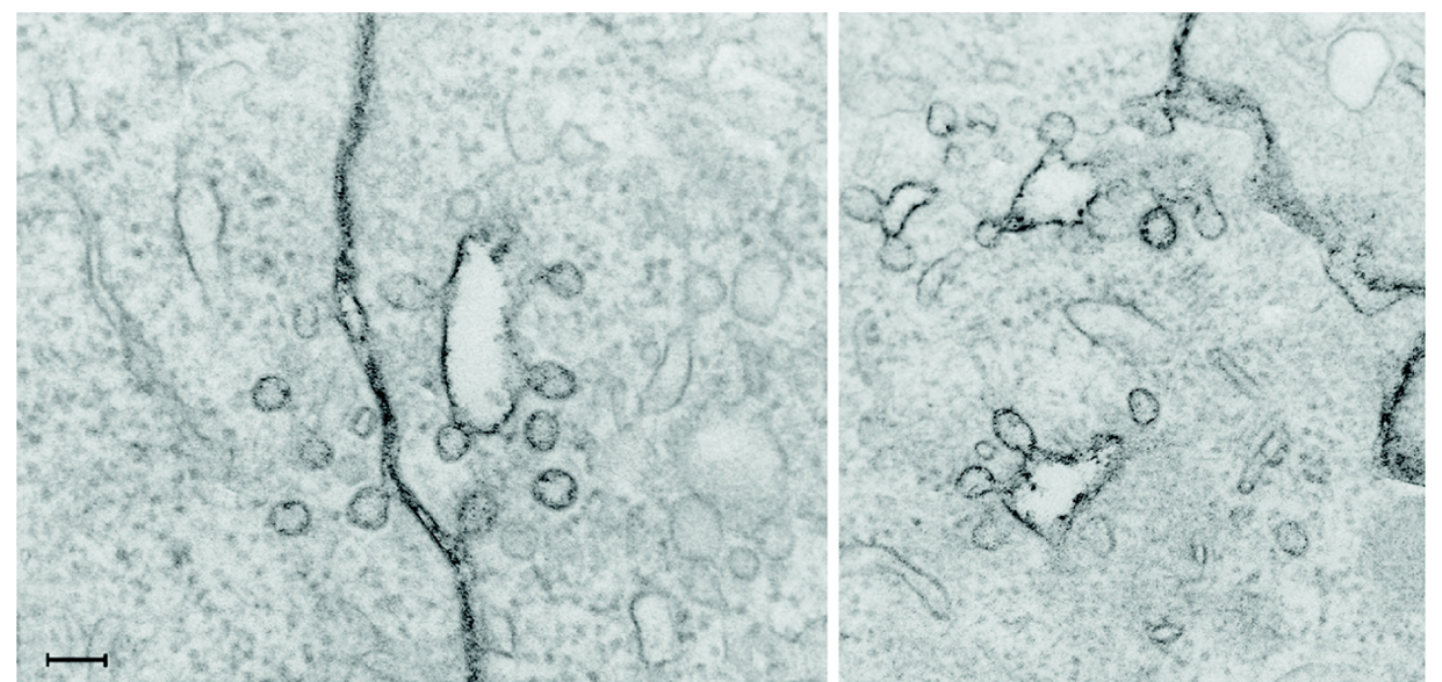

Figure 2. EM images obtained with Ruthenium red added during fixation. The black staining of the plasma membrane reveals that caveolae which may appear to be internalized vesicles in the cytosol, are surface connected. Bar: $100 \mathrm{~nm}$. This figure is reproduced from Current Opinion in Cell Biology (Sandvig et al.: Clathrin-independent endocytosis: mechanism and function) [4] with permission from Elsevier. 


\section{Size of NPs that can be taken up via caveolae}

Based on the information above, it is clear that one needs to be very careful before concluding that NPs are taken up via caveolae. As mentioned above, the flask-formed caveolae typically have a size of 50-80 $\mathrm{nm}$ [8]. We are not aware that any NPs with a size larger than $100 \mathrm{~nm}$ has been unambiguously demonstrated to be taken up via caveolae. Nevertheless, it is concluded in several articles that NPs much larger than $100 \mathrm{~nm}$ are taken up via caveolae. Researchers stating that NPs larger than the size of the caveolae structure are endocytosed via caveolae should be asked to clearly demonstrate their unlikely conclusion, often based on unspecific inhibitors, before that manuscript is accepted for publication. As described above, if NPs are taken up via caveolae, they will first be transported to endosomes similarly as described for uptake via other endocytic mechanisms. These NPs may then follow normal intracellular transport pathways, which include recycling back to the cell surface, retrograde transport to the Golgi apparatus and the endoplasmic reticulum, and transport to lysosomes $[1,26]$.

\section{CONCLUSIONS}

It is remarkable how many articles are still being published where the authors state that the NPs studied are very promising for drug delivery because they are taken up via caveolae and therefore avoid lysosomal degradation. As discussed above, one needs to be very careful when using pharmacological inhibitors to investigate which endocytic mechanisms are involved in the cellular uptake of NPs. The literature shows many examples where the conclusion that the NPs are taken up via caveolae is not supported by the data shown. Thus, conclusions published in many articles about the uptake of NPs via caveolae during the last 15 years should be reevaluated in light of the information given above. It is also very important for the NP community that review articles are not only referring to old statements about the uptake of NPs via caveolae but checking the data behind such conclusions. When authors or reviewers see a description of caveosomes or statements that cellular uptake of NPs larger than the size of caveolae is taken up via these structures of $50-80 \mathrm{~nm}$, then we hope that this short article will make a bell ring.

\section{Acknowledgments}

The authors have been supported by The Norwegian Cancer Society and the Norwegian Research Council in their work related to this article.

\section{Conflict of Interests}

The authors declare no conflicts of interest. For a signed statement, please contact the journal office: editor@precisionnanomedicine.com

Quote this article as Skotland T, Iversen TG, and Sandvig K, Cellular uptake of nanoparticles: Involvement of caveolae, Precis. Nanomed. 2021;4(2):782-786, https://doi.org/10.33218/001c.22201

\section{References}

[1] T.G. Iversen, T. Skotland, K. Sandvig, Endocytosis and intracellular transport of nanoparticles: Present knowledge and need for future studies, Nano Today, 6 (2011) 176-185.

[2] K. Sandvig, S. Kavaliauskiene, T. Skotland, Clathrin-independent endocytosis: an increasing degree of complexity, Histochem Cell Biol, 150 (2018) 107-118.

[3] J.J. Rennick, A.P.R. Johnston, R.G. Parton, Key principles and methods for studying the endocytosis of biological and nanoparticle therapeutics, Nat Nanotechnol, 16 (2021) 266-276.

[4] K. Sandvig, S. Pust, T. Skotland, B. van Deurs, Clathrin-independent endocytosis: mechanisms and function, Curr. Opin. Cell Biol, 23 (2011) 413-420.

[5] Y.J. Erden, R. Lévy, The long life of unicorns, Precis. Nanomed., 3 (2020) 677-683.

[6] B. Fadeel, Comment on "The long life of unicorns," Precis. Nanomed., 3 (2020) 709.

[7] R.G. Parton, M.A. Del Pozo, S. Vassilopoulos, I.R. Nabi, S. Le Lay, R. Lundmark, A.K. Kenworthy,

A. Camus, C.M. Blouin, W.C. Sessa, C. Lamaze, Caveolae: The FAQs, Traffic, 21 (2020) 181-185. 
[8] R.G. Parton, K.A. McMahon, Y. Wu, Caveolae: Formation, dynamics, and function, Current opinion in cell biology, 65 (2020) 8-16.

[9] A.M. Hommelgaard, K. Roepstorff, F. Vilhardt, M.L. Torgersen, K. Sandvig, B. van Deurs, Caveolae: stable membrane domains with a potential for internalization, Traffic, 6 (2005) 720-724.

[10] J.E. Schnitzer, P. Oh, Albondin-mediated capillary permeability to albumin. Differential role of receptors in endothelial transcytosis and endocytosis of native and modified albumins, J Biol Chem, 269 (1994) 6072-6082.

[11] M.J. Hawkins, P. Soon-Shiong, N. Desai, Protein nanoparticles as drug carriers in clinical medicine, Adv Drug Deliv Rev, 60 (2008) 876-885.

[12] B. van Deurs, K. Roepstorff, A.M. Hommelgaard, K. Sandvig, Caveolae: anchored, multifunctional platforms in the lipid ocean, Trends Cell Biol, 13 (2003) 92-100.

[13] L. Pelkmans, J. Kartenbeck, A. Helenius, Caveolar endocytosis of simian virus 40 reveals a new two-step vesicular-transport pathway to the ER, Nat. Cell Biol, 3 (2001) 473-483.

[14] E.M. Damm, L. Pelkmans, J. Kartenbeck, A. Mezzacasa, T. Kurzchalia, A. Helenius, Clathrin- and caveolin-1-independent endocytosis: entry of simian virus 40 into cells devoid of caveolae, J. Cell Biol, 168 (2005) 477-488.

[15] A. Hayer, M. Stoeber, D. Ritz, S. Engel, H.H. Meyer, A. Helenius, Caveolin-1 is ubiquitinated and targeted to intralumenal vesicles in endolysosomes for degradation, J. Cell Biol, 191 (2010) 615-629.

[16] J. Yu, S. Bergaya, T. Murata, I.F. Alp, M.P. Bauer, M.I. Lin, M. Drab, T.V. Kurzchalia, R.V. Stan, W.C. Sessa, Direct evidence for the role of caveolin-1 and caveolae in mechanotransduction and remodeling of blood vessels, J Clin Invest, 116 (2006) 1284-1291.

[17] S. Torrino, W.W. Shen, C.M. Blouin, S.K. Mani, C. Viaris de Lesegno, P. Bost, A. Grassart, D. Koster, C.A. Valades-Cruz, V. Chambon, L. Johannes, P. Pierobon, V. Soumelis, C. Coirault, S. Vassilopoulos, C. Lamaze, EHD2 is a mechanotransducer connecting caveolae dynamics with gene transcription, J Cell Biol, 217 (2018) 4092-4105.

[18] S.K. Rodal, G. Skretting, O. Garred, F. Vilhardt, B. van Deurs, K. Sandvig, Extraction of cholesterol with methyl-beta-cyclodextrin perturbs formation of clathrin-coated endocytic vesicles, Mol. Biol. Cell, 10 (1999) 961-974.

[19] S. Grimmer, B. van Deurs, K. Sandvig, Membrane ruffling and macropinocytosis in A431 cells require cholesterol, J. Cell Sci, 115 (2002) 2953-2962.

[20] G.J. Doherty, H.T. McMahon, Mechanisms of endocytosis, Annu. Rev. Biochem, 78 (2009) 857902.

[21] T. Skotland, S. Kavaliauskiene, K. Sandvig, The role of lipid species in membranes and cancerrelated changes, Cancer Metastasis Rev, 39 (2020) 343-360.

[22] L. Pelkmans, D. Puntener, A. Helenius, Local actin polymerization and dynamin recruitment in SV40-induced internalization of caveolae, Science, 296 (2002) 535-539.

[23] M.L. Torgersen, G. Skretting, B. van Deurs, K. Sandvig, Internalization of cholera toxin by different endocytic mechanisms, J. Cell Sci, 114 (2001) 3737-3747.

[24] H. Shogomori, A.H. Futerman, Cholera toxin is found in detergent-insoluble rafts/domains at the cell surface of hippocampal neurons but is internalized via a raft-independent mechanism, J. Biol. Chem, 276 (2001) 9182-9188.

[25] B.J. Nichols, A.K. Kenworthy, R.S. Polishchuk, R. Lodge, T.H. Roberts, K. Hirschberg, R.D. Phair, J. Lippincott-Schwartz, Rapid cycling of lipid raft markers between the cell surface and Golgi complex, J Cell Biol, 153 (2001) 529-541.

[26] K. Sandvig, T. Skotland, B. van Deurs, T.I. Klokk, Retrograde transport of protein toxins through the Golgi apparatus, Histochem Cell Biol, 140 (2013) 317-326. 